\title{
Effects of White Matter Hyperintensities on 90-Day Functional Outcome after Large Vessel and Non-Large Vessel Stroke
}

\author{
Christoph Johannes Griessenauer ${ }^{a, b}$ David McPherson ${ }^{c}$ Andrea Berger ${ }^{d}$ Ping Cuiper $^{d}$ \\ Nelson Sofoluke ${ }^{a}$ Matthew D. Adams ${ }^{c}$ Saran Kunaprayoon ${ }^{c}$ Ramin Zand ${ }^{e}$ Jiang Li $^{f}$ \\ Vida Abedif,g Oded Goren ${ }^{\mathrm{a}}$ Clemens M. Schirmer ${ }^{\mathrm{a}}$ Kathleen Donahue ${ }^{\mathrm{h}}$ Marco Nardin $^{\mathrm{h}}$ \\ Anne-Karin Giese $^{\mathrm{h}}$ Markus D. Schirmer ${ }^{\mathrm{h}}$ Natalia S. Rost ${ }^{\mathrm{h}} \quad$ Philipp Hendrix $^{\mathrm{a}, \mathrm{i}}$ \\ aDepartment of Neurosurgery, Geisinger, Danville, PA, USA; ${ }^{\mathrm{b}}$ Research Institute of Neurointervention, Paracelsus \\ Medical University, Salzburg, Austria; 'Geisinger Commonwealth School of Medicine, Scranton, PA, USA; \\ 'Biostatistics Core, Geisinger, Danville, PA, USA; 'Department of Neurology, Geisinger, Danville, PA, USA;

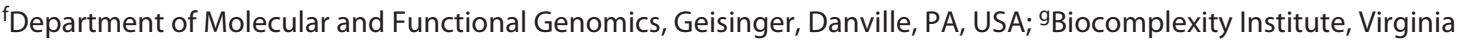 \\ Tech, Blacksburg, VA, USA; ' hepartment of Neurology, Massachusetts General Hospital, Harvard Medical School, \\ Boston, MA, USA; 'Department of Neurosurgery, Saarland University Medical Center and Saarland University Faculty \\ of Medicine, Homburg/Saar, Germany
}

\section{Keywords}

Stroke $\cdot$ White matter $\cdot$ Hyperintensity $\cdot$ Large vessel occlusion - Outcome

\begin{abstract}
Introduction: White matter hyperintensity (WMH) burden is a critically important cerebrovascular phenotype related to the diagnosis and prognosis of acute ischemic stroke. The effect of WMH burden on functional outcome in large vessel occlusion (LVO) stroke has only been sparsely assessed, and direct LVO and non-LVO comparisons are currently lacking. Material and Methods: We reviewed acute ischemic stroke patients admitted between 2009 and 2017 at a large healthcare system in the USA. Patients with LVO were identified and clinical characteristics, including 90-day functional outcomes, were assessed. Clinical brain MRIs obtained at the time of the stroke underwent quantification of WMH using a fully automated algorithm. The pipeline incorporated automated brain extraction, intensity normalization, and $\mathrm{WMH}$
\end{abstract}

\section{KARGER}

(c) 2020 S. Karger AG, Basel

karger@karger.com

www.karger.com/ced segmentation. Results: A total of 1,601 acute ischemic strokes with documented 90-day mRS were identified, including 353 (22\%) with LVO. Among those strokes, WMH volume was available in 1,285 (80.3\%) who had a brain MRI suitable for WMH quantification. Increasing WMH volume from 0 to $4 \mathrm{~mL}$, age, female gender, a number of stroke risk factors, presence of LVO, and higher NIHSS at presentation all decreased the odds for a favorable outcome. Increasing $\mathrm{WMH}$ above $4 \mathrm{~mL}$, however, was not associated with decreasing odds of favorable outcome. While WMH volume was associated with functional outcome in non-LVO stroke ( $p=0.0009)$, this association between WMH and functional status was not statistically significant in the complete case multivariable model of LVO stroke ( $p=0.0637)$. Conclusion: The burden of WMH has effects on 90-day functional outcome after LVO and non-LVO strokes. Particularly, increases from no measurable $\mathrm{WMH}$ to $4 \mathrm{~mL}$ of WMH correlate strongly with the outcome. Whether this relationship of increasing WMH to worse outcome is more pronounced in non-LVO than LVO strokes deserves additional investigation.

(c) 2020 S. Karger AG, Basel
Christoph Johannes Griessenaue

Department of Neurosurgery, Geisinger Health System

$100 \mathrm{~N}$ Academy Ave.

Danville 17822 (USA)

christoph.griessenauer@gmail.com 


\section{Introduction}

White matter hyperintensity (WMH) of vascular origin is a key imaging manifestation of cerebral small vessel disease and associated with stroke and vascular dementia $[1,2]$. The pathophysiologic process by which WMH develops includes endothelial dysfunction, disturbance of the microcirculation, and blood-brain barrier breakdown at the level of cerebral arterioles, capillaries, and venules $[2,3]$. In acute ischemic stroke, WMH burden has been shown to predict functional and cognitive impairment after the stroke [4-6]. Large vessel occlusion (LVO) strokes are distinct from other forms of ischemic stroke as mechanical thrombectomy, a highly effective therapy in this disease category, is not available to other stroke subtypes [7]. There is, however, limited information on the potential differential effects of WMH burden in LVO and non-LVO strokes on 90-day functional outcome.

\section{Methods}

We reviewed a cohort of acute ischemic stroke patients admitted between 2009 and 2017 to a large healthcare system in the USA recorded in the stroke registry, as previously described [8]. The diagnosis of ischemic stroke was based on the neurologic examination and corresponding neuroimaging. The diagnosis of LVO was based on noninvasive imaging (CT or MR angiography of head and neck) and/or digital subtraction angiography. Proximal anterior circulation $\mathrm{LVO}$ was defined as occlusion of the internal carotid artery or the M1 and/or M2 segments of the middle cerebral artery. Clinical brain MRIs obtained at the time of the stroke admission underwent quantification of WMH volume using a fully automated pipeline incorporating automated brain extraction, intensity normalization, and WMH segmentation as previously described. The volume of WMH is reported in $\mathrm{ml}$, not transformed, and analyzed as a continuous variable [9]. Functional outcome was assessed using the modified Rankin Scale (mRS) with mRS 0-2 representing "favorable" and mRS 3-6 representing "unfavorable" functional outcome. Ethics approval was obtained from the local institutional review board, and informed consent was waived.

\section{Statistical Analysis}

The analysis was performed at the stroke encounter level with each individual stroke admission representing 1 encounter. Categorical variables were described using frequencies and percentages

Table 1. Baseline demographic and stroke risk factor characteristics for all stroke encounters $(n=1,601)$

\begin{tabular}{|c|c|c|c|c|}
\hline Age (median $[\mathrm{IQR}]$, mean $\pm \mathrm{SD}$ ), years & $69([58-78], 67.7 \pm 13.4)$ & $67[57-76], 65.6 \pm 13.3$ & $74[63-82], 72.0 \pm 12.7$ & $<0.0001$ \\
\hline Females, $n(\%)$ & $788(49.2)$ & $502(46.7)$ & $286(54.3)$ & 0.0048 \\
\hline History of hypertension, $n(\%)$ & $1,264(79.0)$ & $814(75.8)$ & $450(85.4)$ & $<0.0001$ \\
\hline Diabetes mellitus type $2, n(\%)$ & $621(38.8)$ & $375(34.9)$ & $246(46.7)$ & $<0.0001$ \\
\hline Missing, $n$ & 29 & 11 & 18 & \\
\hline Alcohol consumption, $n(\%)$ & $552(36.5)$ & $407(39.9)$ & $145(29.5)$ & $<0.0001$ \\
\hline Missing, $n$ & 89 & 54 & 35 & \\
\hline $\mathrm{BMI} \geq 30, n(\%)$ & $755(47.2)$ & $508(47.3)$ & $247(46.9)$ & 0.8732 \\
\hline Peripheral vascular disease, $n(\%)$ & $161(10.1)$ & $92(8.6)$ & $69(13.1)$ & 0.0060 \\
\hline Coronary artery disease, $n(\%)$ & $477(29.8)$ & $286(26.6)$ & $191(36.2)$ & $<0.0001$ \\
\hline Procoagulant coagulation disorder, $n(\%)$ & $71(4.4)$ & $42(3.9)$ & $29(5.5)$ & 0.1560 \\
\hline $\mathrm{COPD}, n(\%)$ & $188(11.7)$ & $104(9.7)$ & $84(15.9)$ & 0.0004 \\
\hline Sleep apnea, $n(\%)$ & $170(10.6)$ & $102(9.5)$ & $68(12.9)$ & 0.0468 \\
\hline Family history of stroke, $n(\%)$ & $358(22.4)$ & $224(20.9)$ & $134(25.4)$ & 0.0412 \\
\hline Prior TIA, $n(\%)$ & $191(11.9)$ & $120(11.2)$ & $71(13.5)$ & 0.1897 \\
\hline Prior stroke (hemorrhagic or ischemic), $n(\%)$ & $342(21.4)$ & $200(18.6)$ & $142(26.9)$ & 0.0002 \\
\hline
\end{tabular}

mRS, modified Rankin Scale; IQR, interquartile range. * $p$ value: continuous variables were compared using Wilcoxon rank sum tests, and categorical variables were compared using Fisher's exact tests. 
and continuous variables were described using means and standard deviations, or medians and interquartile ranges. Comparisons of demographic and clinical variables between observations with and without WMH volume and between observations resulting in an mRS of 0-2 (favorable) versus mRS 3-6 (unfavorable) were performed using Fisher's exact tests for categorical variables and Wilcoxon rank sum tests for continuous variables.

Multiple imputations for the missing values was performed using the multivariate imputation by fully conditional specification methods $[10,11]$. Four complete datasets were created. In addition to the 4 variables for which missing values were imputed, 43 additional variables were used to inform the multiple imputation process. A complete list of those variables can be found in Table 1 in the online suppl. only content; for all online suppl. material, see www.karger.com/doi/10.1159/000509071. To facilitate the imputation of missing values of NIHSS, the scores were categorized. A score of 0 was classified as no stroke symptoms, scores of 1-4 indicated minor stroke, scores of 5-15 moderate stroke, scores of 16-20 moderate-to-severe stroke, and scores of 21-42 severe stroke. Results from analyzing each of the 4 complete datasets were combined using methods from Rubin [12]. The procedure computes combined point estimates and combined standard errors, while accounting for within-imputation and between-imputation variance.

The relationship between $\mathrm{WMH}$ volume and the functional status outcomes was described using odds ratio (OR) estimates and 95\% confidence intervals (CIs) from multivariable logistic regression models adjusting for patient and treatment characteristics. Variables that were statistically significant $(p<0.05)$ in bivariate analysis were included in the multivariable regression models. The categorical version of NIHSS at admission was used in multivariable models. Restricted cubic splines were used to test for a nonlinear relationship between WMH volume and the functional status outcome. Multivariable model results are reported for complete cases where no observations had missing values for variables included in the models (complete case analysis), and analysis was repeated on the imputed dataset, and those model results are presented.

Table 2. Imaging, stroke, and treatment characteristics for all encounters $(n=1,601)$

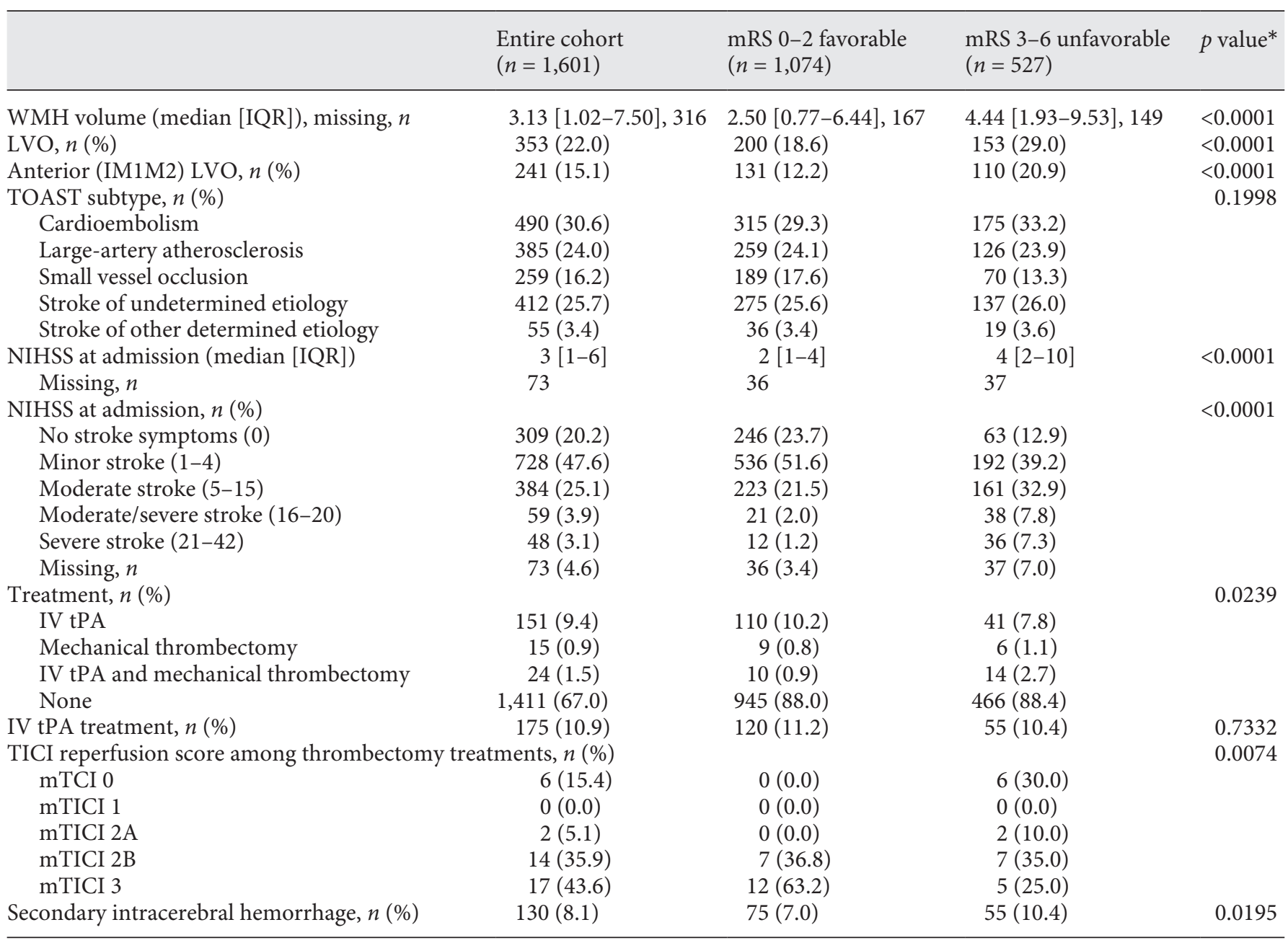

mRS, modified Rankin Scale; WMH, white matter hyperintensity; LVO, large vessel occlusion; IQR, interquartile range. * $p$ value: continuous variables were compared using Wilcoxon rank sum tests, and categorical variables were compared using Fisher's exact tests.

White Matter Hyperintensity in Large Vessel Occlusion Stroke
Cerebrovasc Dis 2020;49:419-426

DOI: $10.1159 / 000509071$ 
Table 3. Complete case analysis multivariable model for favorable functional outcome (all $p<0.05)$

\begin{tabular}{lrrrr}
\hline Effect & OR & 95\% CI & \multicolumn{2}{c}{$p$ value } \\
\hline WMH volume (1 unit increase for volume $\leq 4)$ & 0.863 & 0.767 & 0.972 & 0.0017 \\
WMH volume (1 unit increase for volume $>4)$ & 0.982 & 0.956 & 1.009 & 0.0017 \\
Age & 0.971 & 0.958 & 0.983 & $<0.0001$ \\
Female & 0.699 & 0.526 & 0.930 & 0.0139 \\
Diabetes mellitus type 2 & 0.617 & 0.462 & 0.824 & 0.0011 \\
Peripheral vascular disease & 0.632 & 0.411 & 0.972 & 0.0366 \\
Anemia & 0.448 & 0.310 & 0.647 & $<0.0001$ \\
Sleep apnea & 0.643 & 0.414 & 0.998 & 0.0488 \\
LVO & 0.540 & 0.379 & 0.770 & 0.0007 \\
NIHSS at admission: minor versus no symptoms & 0.895 & 0.606 & 1.321 & $<0.0001$ \\
NIHSS at admission: moderate versus no symptoms & 0.304 & 0.197 & 0.468 & $<0.0001$ \\
NIHSS at admission: moderate/severe versus no symptoms & 0.178 & 0.072 & 0.440 & $<0.0001$ \\
NIHSS at admission: severe versus no symptoms & 0.080 & 0.023 & 0.279 & $<0.0001$ \\
Treatment: mechanical thrombectomy versus none & 17.649 & 1.615 & 192.809 & 0.0077 \\
Treatment: IV tPA versus none & 1.757 & 1.018 & 3.032 & 0.0077 \\
Treatment: IV tPA/Mechanical thrombectomy versus none & 4.837 & 1.095 & 21.372 & 0.0077 \\
\hline
\end{tabular}

OR estimates for complete case analysis $(n=1,171)$. OR, odds ratio; CI, confidence interval; WMH, white matter hyperintensity; LVO, large vessel occlusion.

Table 4. Complete case analysis multivariable model for favorable functional outcome for encounters without LVO (all $p<0.05)$

\begin{tabular}{lcrrr}
\hline Effect & OR & 95\% CI & & $p$ value \\
\hline WMH volume (1 unit increase for volume $\leq 4)$ & 0.841 & 0.732 & 0.966 & 0.0009 \\
WMH volume (1 unit increase for volume $>4)$ & 0.979 & 0.951 & 1.007 & 0.0009 \\
Age & 0.972 & 0.958 & 0.987 & 0.0002 \\
Female & 0.664 & 0.480 & 0.918 & 0.0133 \\
Diabetes mellitus type 2 & 0.606 & 0.436 & 0.842 & 0.0029 \\
Peripheral vascular disease & 0.524 & 0.315 & 0.871 & 0.0126 \\
Carotid stenosis & 1.526 & 1.078 & 2.160 & 0.0171 \\
Anemia & 0.546 & 0.367 & 0.814 & 0.0030 \\
Sleep apnea & 0.601 & 0.373 & 0.968 & 0.0362 \\
NIHSS at admission: minor versus no symptoms & 1.052 & 0.690 & 1.605 & $<0.0001$ \\
NIHSS at admission: moderate versus no symptoms & 0.352 & 0.221 & 0.561 & $<0.0001$ \\
NIHSS at admission: moderate/severe versus no symptoms & 0.111 & 0.022 & 0.549 & $<0.0001$ \\
NIHSS at admission: severe versus no symptoms & 0.324 & 0.056 & 1.871 & $<0.0001$ \\
\hline
\end{tabular}

OR estimates for complete case analysis among non-LVO encounters $(n=927)$. OR, odds ratio; CI, confidence interval; WMH, white matter hyperintensity; LVO, large vessel occlusion.

Exploration of an optimal cutoff value of WMH volume to predict a favorable functional status outcome was done using SAS's ROCPLOT macro from http://support.sas.com/kb/25/018. html\#ref. Plots of the receiver operating characteristic curve associated with the clinically favorable (or unfavorable) binary outcome were produced. All analyses were performed using SAS 9.4 (SAS Institute Inc., Cary, NC, USA).

\section{Results}

Between 2009 and 2017, 1,654 acute ischemic stroke encounters were studied. Fifty-three observations were missing the 90-day $\mathrm{mRS}$ and excluded, resulting in 1,601 stroke encounters in 1,433 unique patients. A total of 316 strokes (19.7\%) were missing WMH volume values. Either no brain 
MRI had been obtained during the encounter or brain MRIs were not suitable for WMH segmentation. Encounters with and without values of WMH were compared and are presented in Table 2 in the online suppl. only content.

\section{Functional Outcome at 90 Days in the Entire Cohort}

Of 1,601 strokes, 1,074 (67.1\%) had a favorable clinical outcome (mRS 0-2), while 527 (32.9\%) had unfavorable outcome (mRS 3-6). Baseline demographics and stroke risk factor characteristics of the 2 outcome groups are shown in Table 1. Imaging, stroke, and treatment characteristics are presented in Table 2. Median WMH volume was lower in patients with favorable outcome ( 2.5 vs. 4.4, $p<0.0001)$. Those with favorable outcomes had lower proportions of LVO (18.6 vs. $29.0 \%, p<0.0001)$ and a smaller proportion of anterior LVO (12.2 vs. $20.9 \%, p<$ 0.0001 ). Strokes resulting in favorable 90-day outcome had lower NIHSS at admission (median of 2 vs. $4, p<$ 0.0001 ). A comparison of categorical values of NIHSS also showed a significant difference $(p<0.0001)$. Encounters with favorable outcomes had a lower proportion of secondary intracerebral hemorrhage ( 7.0 vs. $10.4 \%, p=$ $0.0195)$. Treatment types differed between the 2 groups $(p=0.0239)$. Similar analysis was carried out after excluding 316 observations without values of WMH (Tables 3, 4 in the online suppl. only content). After controlling for patient and treatment characteristics in complete case multivariable models, it was observed that increases in $\mathrm{WMH}$ volumes from 0 to $4 \mathrm{~mL}$ were associated with lower odds of a favorable outcome (OR 0.863, 95\% CI: 0.767, 0.972). However, increases for WMH greater than $4 \mathrm{~mL}$ was not associated with additional decreased odds of a favorable outcome (OR 0.982, 95\% CI: 0.956, 1.009). Thus, the risk of an unfavorable outcome was higher as WMH increased from 0 to $4 \mathrm{~mL}$, but there was no additional risk for $\mathrm{WMH}$ volume with increases above $4 \mathrm{~mL}$ (Table 3; Fig. 1). Performing multivariable analysis on a dataset with imputed values for variables with missing data showed similar results (Table 5 in the online suppl. only content).

\section{Functional Outcome at 90 Days in Non-LVO Ischemic} Strokes

Baseline demographic, stroke risk factor, imaging, stroke, and treatment characteristics are presented in Tables 6 and 7 in the online suppl. only content. After controlling for other patient and treatment characteristics in complete case multivariable models on encounters without LVO, it was observed that increases in $\mathrm{WMH}$ volumes from 0 to $4 \mathrm{~mL}$ were associated with lower odds of a favorable outcome (OR 0.841, 95\% CI: 0.732, 0.966). How-

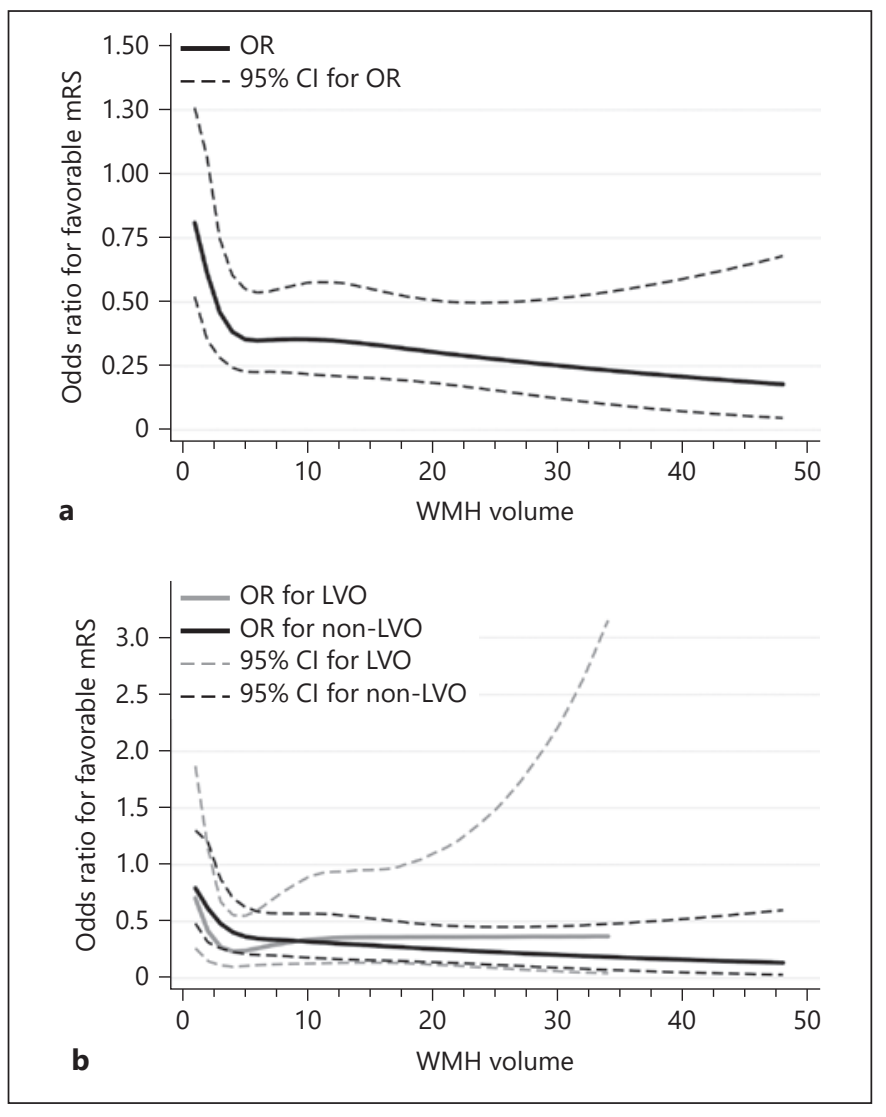

Fig. 1. a WMH and OR estimates for favorable functional status. Estimates are from a logistic regression model using a restricted cubic spline for the WMH variable. ORs (continuous line) and their 95\% CIs (dashed lines) were estimated at each integer value of WMH versus 0 and plotted for WMH ranging from 1 to 48 . Data use observations with non-missing values of WMH $(n=1,285)$. b WMH and OR estimates for favorable functional status for nonLVO $(n=1,005)$ and LVO $(n=280)$ ischemic strokes. OR, odds ratio; $\mathrm{CI}$, confidence interval; $\mathrm{WMH}$, white matter hyperintensity; LVO, large vessel occlusion.

ever, increases for WMH greater than $4 \mathrm{~mL}$ was not associated with additional decreased odds of a favorable outcome (OR 0.979, 95\% CI: 0.951, 1.007) (Table 4). Performing multivariable analysis on a dataset with imputed values for variables with missing data showed similar results (Table 8 in the online suppl. only content).

\section{Functional Outcome at 90 Days in LVO Ischemic Strokes}

Baseline demographic, stroke risk factor, imaging, stroke, and treatment characteristics are presented in Tables 9 and 10 in the online suppl. only content. After controlling for other patient and treatment characteristics in complete case multivariable models on encounters with 
Table 5. Complete case analysis multivariable model for favorable functional outcome for encounters with LVO $($ all $p<0.05)$

\begin{tabular}{lcccc}
\hline Effect & OR & 95\% CI & & $p$ value \\
\hline WMH volume (1 unit increase for volume $\leq 4)$ & 0.761 & 0.604 & 0.960 & 0.0637 \\
WMH volume (1 unit increase for volume >4) & 1.057 & 0.980 & 1.140 & 0.0637 \\
Age & 0.965 & 0.939 & 0.992 & 0.0103 \\
Dyslipidemia & 0.427 & 0.211 & 0.864 & 0.0179 \\
Smoking ever versus never & 2.138 & 1.117 & 4.093 & 0.0218 \\
Anemia & 0.178 & 0.064 & 0.495 & 0.0009 \\
NIHSS at admission: minor versus no symptoms & 0.485 & 0.171 & 1.374 & 0.0067 \\
NIHSS at admission: moderate versus no symptoms & 0.213 & 0.074 & 0.611 & 0.0067 \\
NIHSS at admission: moderate/severe versus no symptoms & 0.247 & 0.067 & 0.913 & 0.0067 \\
NIHSS at admission: severe versus no symptoms & 0.085 & 0.013 & 0.534 & 0.0067 \\
\hline
\end{tabular}

OR estimates for complete case analysis among LVO encounters $(n=255)$. OR, odds ratio; CI, confidence interval; LVO, large vessel occlusion; WMH, white matter hyperintensity.
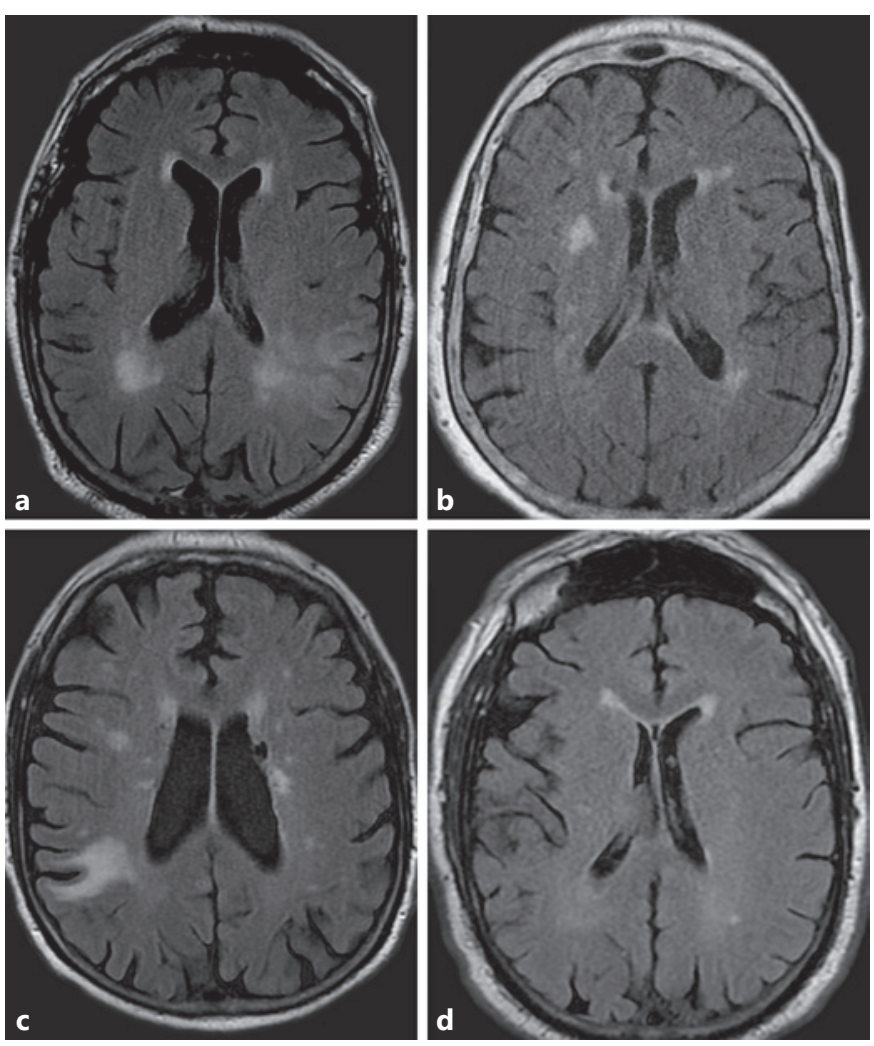

Fig. 2. Representative axial T2 FLAIR MRI brains of strokes with $\mathrm{WMH}$ volumes of approximately $4 \mathrm{~mL}$. a $4.05 \mathrm{~mL}$. b $4.02 \mathrm{~mL}$. c $4.04 \mathrm{~mL}$. d $4.01 \mathrm{~mL}$. WMH, white matter hyperintensity.

LVO, the association between $\mathrm{WMH}$ volume and favorable functional status was not statistically significant $(p=$ 0.0637 ) (Table 5). Unlike the complete case analysis, pro- ducing multivariable models for the larger LVO dataset with imputed values showed a statistically significant association between $\mathrm{WMH}$ and functional status ( $p=$ 0.0065 ) (Table 11 in the online suppl. only content).

\section{Discussion}

The current study assessed associations of WMH burden with 90-day functional outcome after non-LVO and LVO acute ischemic strokes. There was a strong relationship between WMH burden and functional outcome in patients with WMH volumes between 0 and $4 \mathrm{~mL}$ (stroke examples with WMH volumes of approximately $4 \mathrm{~mL}$ are shown in Fig. 2). Patients in this group had decreasing odds for a favorable outcome with increasing WMH. Interestingly, in patients with $\mathrm{WMH}$ above 4 , the relationship between increasing WMH and decreasing odds for a favorable outcome was no longer statistically significant, indicating a threshold effect of WMH. Patients with WMH surpassing that threshold had uniformly unfavorable outcome, independent of the amount of additional $\mathrm{WMH}$. In terms of LVO status, WMH volume was significantly related to functional outcomes in non-LVO strokes. In the complete case multivariable model for LVO stroke (i.e., without imputation for missing variables), the association between WMH volume and functional outcome was not significant. After imputation, however, the effect of WMH volume on functional outcome in LVO strokes was also observed, likely due to increase in statistical power of the imputed dataset analysis. From a clinical point of view, the current study does not demonstrate re- 
markably different effects of WMH burden on functional outcomes in non-LVO and LVO ischemic strokes.

\section{WMH in LVO Ischemic Strokes}

In the present study, increasing $\mathrm{WMH}$ volumes up to $4 \mathrm{~mL}$ were significantly associated with functional outcome at 90 days after imputation. Other factors associated with unfavorable outcome were advanced age, dyslipidemia, anemia, smoking, anemia, COPD, and a family history of stroke. Smoking had a protective effect. The "smoking paradox" on ischemic stroke outcome, however, was not confirmed in a recent meta-analysis [13]. As expected, increasing stroke severity in terms of NIHSS on admission was associated with worse outcome at 90 days.

Mechanical thrombectomy represents a milestone in the management of acute ischemic strokes from LVO [7]. Data on $\mathrm{WMH}$ in patients undergoing mechanical thrombectomy for LVO stroke are sparse and controversial [14]. An early study from 2012 assessed 292 patients who underwent intra-arterial thrombolysis and found that white matter lesions using the semiquantitative Scheltens and Fazekas scores inversely correlated with favorable outcome, survival, and successful recanalization [15]. Recanalization techniques and devices, however, have advanced significantly and intra-arterial thrombolysis has fallen out of favor limiting the applicability of this study to current day management of LVO stroke patients. A more recent study from 2014, but prior to publication of the landmark LVO stroke trials in 2015 [7], also found an association of severe WMH burden and unfavorable outcome at 90 days [16]. In contrast, a study of 73 patients with LVO undergoing mechanical thrombectomy demonstrated that increasing WMH reduced the odds of good collateral grade, but did not affect long term functional outcome [17]. Others have reported similar small vessel disease burden associations with collaterals [18]. A study by Eker et al. [19] did not, however, find a correlation of small vessel disease burden with collaterals in anterior circulation LVO strokes. More recent studies have shed additional light on the WMH burden issue in LVO strokes. A recent study of 496 patients enrolled in the Thrombectomie des Artères Cérébrales (THRACE) trial and 2 prospective cohorts treated with mechanical thrombectomy found that patients with higher WMH burden had safety and efficacy profiles of thrombectomy similar to those in patients with lower WMH burden, but are at higher risk of unfavorable outcome [20]. The study used similar WMH quantification methodology as the present study. Contrasting this method, another recent study published in 2019 used the semiquantitative van Swieten scale to measure $\mathrm{WMH}$ and dichotomized patients to absent-to-mild (van

White Matter Hyperintensity in Large

Vessel Occlusion Stroke
Swieten scale score $0-2$ ) versus moderate-to-severe (3-4). A total of 144 consecutive patients with successful (TICI $\geq$ $2 \mathrm{~b} / 3$ ) mechanical thrombectomy for anterior circulation LVO within $24 \mathrm{~h}$ were included. WMH was independently associated with the onset-to-reperfusion time. The association between onset-to-reperfusion time and the 90-day outcome depended on the degree of WMH burden. Preexisting $\mathrm{WMH}$ was associated with the 90-day functional outcome after successful reperfusion. Patients with a higher WMH burden needed to present earlier than those with lower $\mathrm{WMH}$ to achieve comparable favorable outcomes [21].

Even if increasing WMH burden is associated with an increased risk of an unfavorable functional outcome, there is no reason to exclude patients with WMH and LVO from receiving this intervention. Mechanical thrombectomy performed both in the early ( $<6-8 \mathrm{~h}$ ) and late (up to $16-24 \mathrm{~h}$ ) time window from stroke onset is beneficial across a wide range of stroke patient characteristics. And while some characteristics (longer time to intervention, advanced age, and higher NIHSS strokes) are associated with worse outcomes than others, the benefit of thrombectomy over medical therapy remains robust across the board [7, 22].

\section{Limitations}

Data collection and analysis were performed retrospectively and were, therefore, subject to incomplete datasets. To mitigate this limitation, imputation for missing variables was performed. For $19.7 \%$ of strokes, no brain MRIs were available for segmentation. Those patients were older, had higher rates of certain stroke risk factors and comorbidities, and had worse functional outcome (Table 2 in the online suppl. only content). A certain selection bias affecting the results of the study can, therefore, not be excluded. The study also included patients with ischemic stroke presenting prior to 2015, at which time the benefit of mechanical thrombectomy for LVO stroke had not been proven. Therefore, mechanical thrombectomy was not offered to all LVO patients eligible for this procedure under current guidelines [23].

\section{Conclusion}

The burden of WMH has effects on 90-day functional outcome after LVO and non-LVO strokes. Particularly increases from no measurable WMH to $4 \mathrm{~mL}$ of WMH correlate strongly with unfavorable outcome. Whether this relationship of increasing WMH to worse outcome is more pronounced in non-LVO than LVO strokes deserves additional investigation. 


\section{Statement of Ethics}

The study protocol was approved by the institutional review board (IRB) at Geisinger (IRB \#: 2017-0521).

\section{Conflict of Interest Statement}

The authors have no conflicts of interest to declare.

\section{Author Contributions}

Conception and design: C.G., D.P., A.B., N.R., and P.H. Acquisition of 3: C.G., D.M., N.S., M.A., S.K., K.D., M.N., A.G., and M.S. Analysis and interpretation of data: C.G., A.B., P.C., J.L., V.A., O.G., C.S., N.R., and P.H. Drafting the article: C.G., D.P., and A.B. Critically revising the article: all authors. Reviewed submitted version of manuscript: all authors. Approval of the final version of the manuscript on behalf of all authors: C.G. Statistical analysis: A.B., P.C., J.L., and V.A. Administrative/technical/material support: C.G., A.B., N.S., and P.H. Study supervision: C.G., N.R., and P.H.

\section{Funding Sources}

This research was supported by NIH grant R01NS086905 (MRI-genetics interface exploration MRI-GENIE study) to Dr. Natalia Rost as the principal investigator.

\section{References}

1 Debette S, Schilling S, Duperron M-G, Larsson SC, Markus HS. Clinical significance of magnetic resonance imaging markers of vascular brain injury: a systematic review and meta-analysis. JAMA Neurol. 2019 Jan;76(1): 81-94.

2 Wardlaw JM, Smith C, Dichgans M. Small vessel disease: mechanisms and clinical implications. Lancet Neurol. 2019 Jul;18(7):68496.

3 Fladt J, Kronlage C, De Marchis GM. Cerebral white matter hyperintensities and microbleeds in acute ischemic stroke: impact on recanalization therapies. A review of the literature. Neurosci Lett. 2018 20;687:55-64.

4 Molad J, Hallevi H, Korczyn AD, Kliper E, Auriel E, Bornstein NM, et al. Vascular and neurodegenerative markers for the prediction of post-stroke cognitive impairment: results from the TABASCO study. J Alzheimers Dis JAD. 2019 Jul;70(3):889-98.

5 Rost NS, Cougo P, Lorenzano S, Li H, Cloonan L, Bouts MJ, et al. Diffuse microvascular dysfunction and loss of white matter integrity predict poor outcomes in patients with acute ischemic stroke. J Cereb Blood Flow Metab. 2018;38(1):75-86.

6 Zamboni G, Griffanti L, Mazzucco S, Pendlebury ST, Rothwell PM. Age-dependent association of white matter abnormality with cognition after TIA or minor stroke. Neurology. 2019 Jul;93(3):e272-82.

7 Goyal M, Menon BK, van Zwam WH, Dippel DWJ, Mitchell PJ, Demchuk AM, et al. Endovascular thrombectomy after large-vessel ischaemic stroke: a meta-analysis of individual patient data from five randomised trials. Lancet Lond Engl. 2016 Apr;387(10029): 1723-31.

8 Hendrix P, Sofoluke N, Adams MD, Kunaprayoon S, Zand R, Kolinovsky AN, et al.
Risk factors for acute ischemic stroke caused by anterior large vessel occlusion. Stroke. 2019 May;50(5):1074-80.

9 Schirmer MD, Dalca AV, Sridharan R, Giese A-K, Donahue KL, Nardin MJ, et al. White matter hyperintensity quantification in largescale clinical acute ischemic stroke cohorts: the MRI-GENIE study. NeuroImage Clin. 2019 May;23:101884.

10 Brand JPL. Development, mplementation and evaluation of multiple imputation strategies for the statistical analysis of incomplete data sets. 1999

11 van Buuren S. Multiple imputation of discrete and continuous data by fully conditional specification. Stat Methods Med Res. 2007 Jun;16(3):219-42.

12 Rubin DB. Multiple imputation for nonresponse in surveys. New York: John Wiley \& Sons; 1987.

13 Li B, Li D, Liu J-F, Wang L, Li B-Z, Yan X-J, et al. "Smoking paradox" is not true in patients with ischemic stroke: a systematic review and meta-analysis. J Neurol. 2019 Oct. Online ahead of print.

14 Mechtouff L, Nighoghossian N, Amaz C, Buisson $\mathrm{M}$, Berthezène $\mathrm{Y}$, Derex $\mathrm{L}$, et al. White matter burden does not influence the outcome of mechanical thrombectomy. J Neurol. 2020 Mar;267(3):618-24.

15 Jung S, Mono ML, Findling O, Fischer U, Galimanis A, Weck A, et al. White matter lesions and intra-arterial thrombolysis. J Neurol. 2012 Jul;259(7):1331-6.

16 Zhang J, Puri AS, Khan MA, Goddeau RP, Henninger N. Leukoaraiosis predicts a poor 90-day outcome after endovascular stroke therapy. AJNR Am J Neuroradiol. 2014 Dec; 35(11):2070-5.

17 Giurgiutiu D-V, Yoo AJ, Fitzpatrick K, Chaudhry Z, Leslie-Mazwi T, Schwamm LH, et al. Severity of leukoaraiosis, leptomeningeal collaterals, and clinical outcomes after intra-arterial therapy in patients with acute ischemic stroke. J Neurointerventional Surg. 2015 May;7(5):326-30.

18 Lin MP, Brott TG, Liebeskind DS, Meschia JF, Sam K, Gottesman RF. Collateral recruitment is impaired by cerebral small vessel disease. Stroke. 2020 May;51(5):1404-10.

19 Eker OF, Rascle L, Cho T-H, Mechtouff L, Derex L, Ong E, et al. Does small vessel disease burden impact collateral circulation in ischemic stroke treated by mechanical thrombectomy? Stroke. 2019;50(6):1582-5.

20 Boulouis G, Bricout N, Benhassen W, Ferrigno $\mathrm{M}$, Turc G, Bretzner $\mathrm{M}$, et al. White matter hyperintensity burden in patients with ischemic stroke treated with thrombectomy. Neurology. 2019 Oct;93(16):e1498-506.

21 Mikati AG, Mandelbaum M, Sapnar S, Puri AS, Silver B, Goddeau RP, et al. Impact of leukoaraiosis severity on the association of time to successful reperfusion with 90 -day functional outcome after large vessel occlusion stroke. Transl Stroke Res. 2020 Feb;11(1):3949.

22 Lansberg MG, Mlynash M, Hamilton S, Yeatts SD, Christensen S, Kemp S, et al. Association of thrombectomy with stroke outcomes among patient subgroups: secondary analyses of the DEFUSE 3 randomized clinical trial. JAMA Neurol. 2019 Apr;76(4):447-53.

23 Powers WJ, Rabinstein AA, Ackerson T, Adeoye OM, Bambakidis NC, Becker K, et al. Guidelines for the early management of patients with acute ischemic stroke: 2019 update to the 2018 guidelines for the early management of acute ischemic stroke: a guideline for healthcare professionals from the American Heart Association/American Stroke Association. Stroke. 2019 Dec;50(12):e344-418. 